\title{
Recent trends in the electroanalytical detection of food fraud
}

\begin{abstract}
Food fraud is a criminal act that has continuously increased in the recent years and represents an important issue to consumers' confidence, which encompass economic, social, health and religious aspects. Electrochemical sensors are a promising category of analytical techniques for food authenticity assessment due to their low cost, simplicity/ easiness-to-perform, sensitivity and sensibility in comparison to other consolidated assays. Herein, a background on the transduction mechanisms of potentiometric, amperometric and impedimetric sensors is provided in addition to some current applications on the authentication of food products.
\end{abstract}

Keywords: food fraud, authentication, electrochemical sensors, analytical techniques
Volume 5 Issue $3-2019$

\author{
Ricardo Adriano Dorledo de Faria, ${ }^{1,2}$ Luiz \\ Guilherme Dias Heneine, ${ }^{3}$ Tulio Matencio, ${ }^{4}$ \\ Younès Messaddeq ${ }^{2,5}$ \\ 'Department of Chemical Engineering, Universidade Federal de \\ Minas Gerais, Brazil \\ ${ }^{2}$ Center for Optics, Photonics and Lasers (COPL), Université \\ Laval, Canada \\ ${ }^{3}$ Department of Applied Immunology, Fundação Ezequiel Dias, \\ Brazil \\ ${ }^{4}$ Department of Chemistry, Universidade Federal de Minas \\ Gerais, Brazil \\ ${ }^{5}$ UNESP, Brazil
}

\begin{abstract}
Correspondence: Ricardo Adriano Dorledo de Faria, Center for Optics, Photonics and Lasers (COPL), Pavillon d'optiquephotonique, Université Laval, 2375 rue de la Terrasse, Quebec, Canada, Tel (4I8) 656 2I3I (Ext 7l84), Email ricardo.adriano08@hotmail.com
\end{abstract}

Received: April 29, 2019 | Published: May 09, 2019

\section{Introduction}

Significant focus towards the quality standard of the food industry reflects consumers increasing concern about food fraud and the authenticity and quality of what they eat. The general term "food fraud" corresponds to the act of substituting, adding or adulterating a food product in order to obtain economic gain. ${ }^{1}$ It differs from the simple definition of "contamination" because the former necessarily relates to an intentional activity. ${ }^{2}$ The Grocery Manufacturers Association (GMA) complements that these criminal acts are carried out by "unapproved enhancements, dilution with a lesser-value ingredient, concealment of damage or contamination, mislabeling of a product or ingredient, substitution of a lesser-value ingredient or failing to disclose required product information" 3 The economically motivated adulteration represents to the North American industry an average cost of 10-15 billion dollars per year and some studies estimate that approximately $10 \%$ of the shelf products in the market still contain some adulteration. ${ }^{3}$ Furthermore, this activity involves the use of a lower valued ingredient at the place of a higher valued one, consisting as a potential disrupting agent of the economy that stimulates the unfair market competition at regional and global levels. ${ }^{4}$ From the consumers' point-of-view, although it is usually not directly associated to health risks, this may pose a threat to allergic population, in addition to go against some religious, social and life-style aspects. ${ }^{5}$ In the light of the criminal nature of fraud, one must takes in account the factors that leads a company to commit this act in order to preserve the consumer's rights. According to the routine activity theory of Cohen and Felson, ${ }^{6}$ a crime happens when at a certain moment and place the following three aspects converge: (i) the presence of motivated agents to commit the crime, (ii) suitable targets and (iii) the absence of agents capable to avoid it. These authors emphasize that the absence of only one of these components is enough to prevent the crime occurrence. In this sense, measures of fraud control takes a crucial place in counteracting the fraud vulnerability by diminishing the possibilities of offenders to find opportunities in committing the crime. ${ }^{7}$ Despite the food fraud phenomenon does not demerit exclusively the current age since there are some historical reports even from the Roman and Greek Empires, it has received more attention in the recent years with the advent and development of more technological methods to detect the presence of fraudulent ingredients in food products. ${ }^{8}$ After a bibliographic search in the Web of Science database for the keywords "food fraud" (Figure 1), it becomes evident the importance of this issue especially in this decade. It surely gained force with the outcome of fraud scandals worldwide, such as the presence of melamine in Chinese milk in $2008^{9}$ and the incident involving horsemeat in Europe in $2013 .{ }^{10} \mathrm{In}$ response to the increasing concern on food authenticity, the scientific community has devoted great attention to develop accurate methods to detect the presence of fraudulent components in food products. The main studied techniques nowadays contemplates physical, chemical, biochemical and molecular transductions. Sheika ${ }^{11}$ mentions that the physical techniques consist on the microscopic/macroscopic analysis of the food structure, the chemical and biochemical methods comprise mainly chromatographies, spectroscopies, immunologic and electrophoretic techniques and the molecular transduction involves the use of DNA-based analysis. According to Danezis et al. ${ }^{12}$ chromatographic and molecular methods are present in almost half of the published articles related to food authentication. However, among all these possibilities, the electrochemical sensors stand out as a promising tool for the detection of food fraud. The mechanism of recognition of these sensors rely on the conversion of the input signal (analyte recognition) into an electrical output signal that is proportional to the concentration of the target molecule. Depending on the type of measured output signal (e.g. voltage, current, impedance), the electrochemical devices can be categorized mainly into potentiometric, amperometric and impedimetric sensors. ${ }^{13}$ Some authors defend that the electrochemical devices can be more sensitive, 
cheap, amenable for in situ and point-of-care applications (due to the possibility of miniaturization of the electrodes), besides they can require small volumes of sample. ${ }^{14-16}$

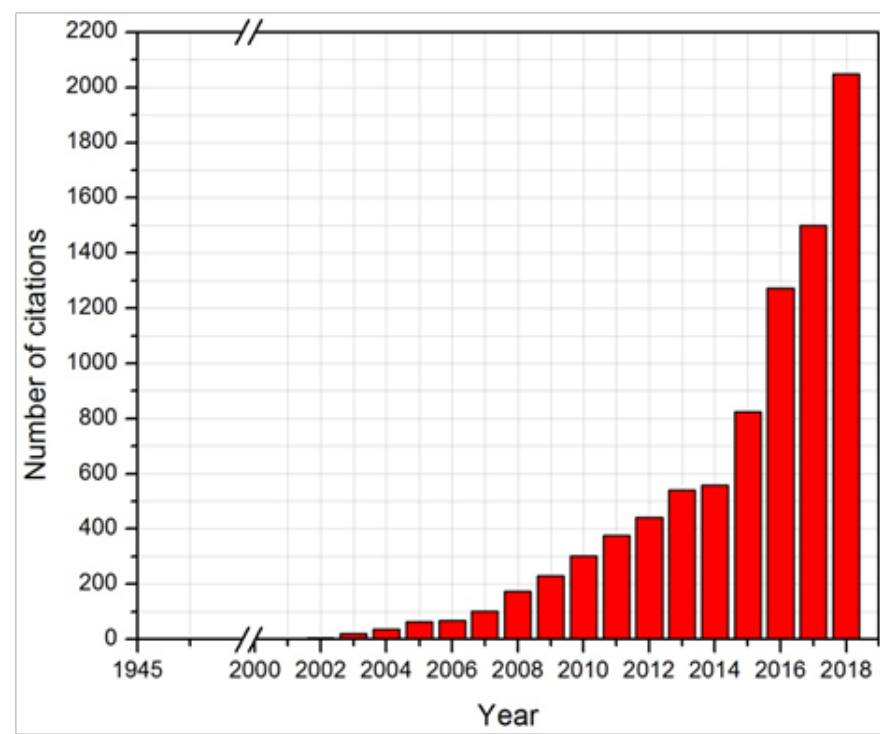

Figure I Evolution of the number of citations of articles containing "food fraud" as a keyword according to the Web of Science database (from 1945 to 2018 ).

\section{Potentiometric sensors}

The working principle of potentiometric sensors consist on measuring the difference in the potential between the working and reference electrodes in the electrochemical cell at the open circuit. This potential can be calculated from the Nernst equation in which the cell potential "E" refers to the value at zero current (electromotive force). ${ }^{16}$ In the equation, "E " refers to the standard potential of the electrode $(\mathrm{V})$, " $\mathrm{R}$ " is the universal gas constant $\left(8.314 \mathrm{~J} . \mathrm{K}^{-1}\right)$, "T" is the

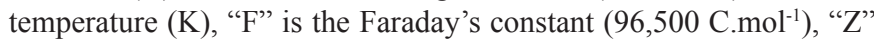
corresponds to the valence of the ion, " $\mathrm{a}_{i}$ " is the activity of the species present in the sample. ${ }^{17}$

$$
E=E_{0}+\frac{R T}{Z F} \ln a_{i}
$$

A deeper interpretation of the Nernst equation leads to the inference about some features of the potentiometric sensors. As notable from the equation, there is a primary dependence between the potential and the logarithm of the ion activity. Since "R" and "F" are constants, the slope of the curve, which relates the sensitivity of the device, depends mainly on the temperature and on the valence of the ion. Thus, in order to achieve proper reproducibility of the sensing response, it is necessary to keep fixed the temperature; otherwise, small changes on it could affect the sensitivity of the sensor (which can represent a challenge for in-situ applications) as seen in Figure 2. For example, considering a potentiometric sensor exposed to a variation of $10 \%$ of the activity of magnesium cations at $298 \mathrm{~K}$, a variation of only $1.2 \mathrm{mV}$ would be expected. Thus, only high variations of the analyte concentration are expected to provide a significant variation of the potential. Figure 2 also shows how the charge of the analyte of interest affects the output potential. Considering, three hypothetical contaminating ions in a food product with valences $Z=1, Z=2$ and $\mathrm{Z}=3\left(\mathrm{~K}^{+}, \mathrm{Mg}^{2+}\right.$ and $\mathrm{Fe}^{3+}$ for instance), it is possible to observe that the ion with the higher value of $\mathrm{Z}$ is subjected to the lowest variations of the potential, which would provide the lower sensitivity of the sensor towards this analyte. Concerning the use of this technology in the food fraud inspection, Trivedi et al., ${ }^{18}$ developed a potentiometric sensor for identification of milk adulteration with urea by means of the sensitive detection of $\mathrm{NH}_{4}^{+}$cations by an ion sensitive membrane containing urease enzyme. A significant amount of articles devoted to the development of potentiometric sensors for food authentication present the technology of electronic tongues for the transduction sensing. The electronic tongues are an instrument composed by a sensor array, and the systems of data acquisition and pattern identification to perform measurements in liquid samples. ${ }^{19}$ Lvova et al., ${ }^{20}$ tested an electronic tongue to investigate wine authenticity, pointing out not only the simplicity of potentiometry as a transduction technique but also its low cost, quickness and the possibility of monitoring plenty of parameters at the same time. In the context of the high number of reports denouncing olive oils fraud, Dias et al., ${ }^{21}$ described the results obtained with a potentiometric electronic tongue for the authentication of extra virgin olive oils, achieving high sensitivity and the capability to distinguish samples from different origins. In comparison to the amperometric sensors, the potentiometric transduction possess as an advantage the characteristic to be non-destructive once the reactants from the bulk solution are not consumed during the analyte recognition, thus, it develops no concentration gradients at the interface with the electrode making it easier to be used..$^{22}$
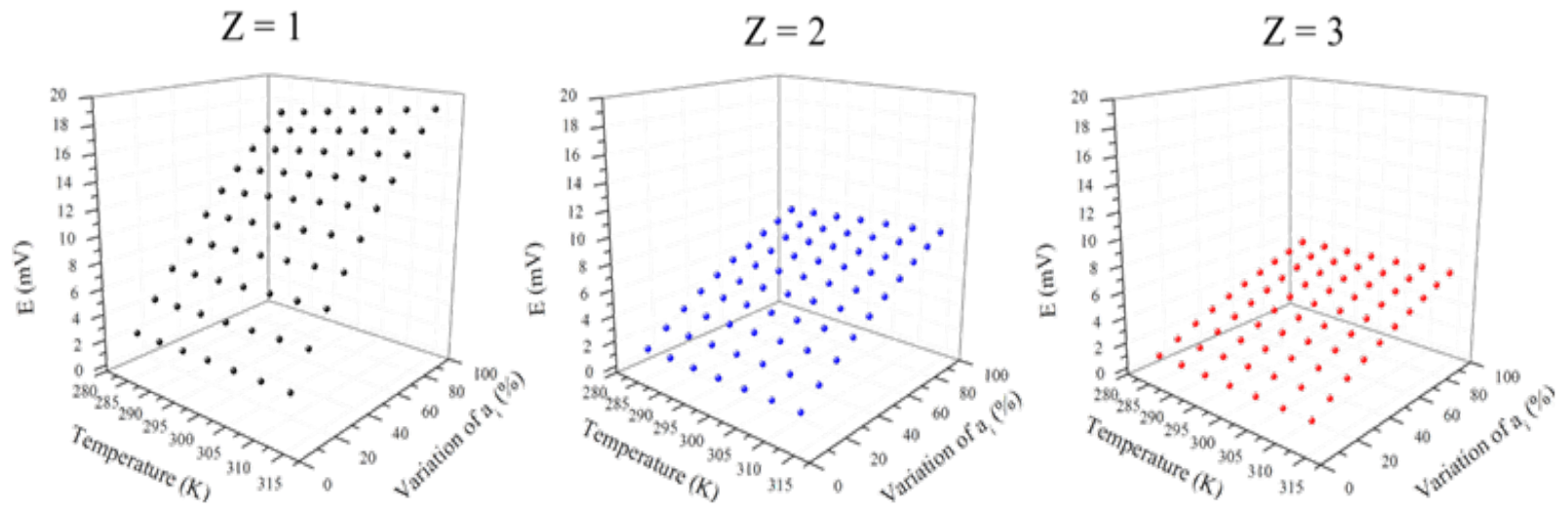

Figure 2 Influence of the temperature, valence of the target ion and the variation of its activity on the expected potential and sensitivity of potentiometric sensors. 


\section{Amperometric sensors}

The amperometry sensing is a technique in which the electrical current developed from reductive and oxidative reactions is measured and interpreted in response to the analyte recognition. A fixed potential is applied to the transducer electrode to favor the redox reactions of the electroactive species from the bulk solution to the interface with the electrolyte. ${ }^{23}$ Until today, the most famous amperometric sensor has been the glucose sensor firstly described by Clark and Lyons in $1962 .{ }^{24}$ The mechanism of transduction was based on the oxidation of glucose mediated by the enzyme glucose oxidase into hydrogen peroxidase. Finally, the hydrogen peroxidase is oxidized to produce free electrons (according to the reaction bellow) that will be measured as a current signal directly proportional to the glucose concentration.

$$
\mathrm{H}_{2} \mathrm{O}_{2} \rightarrow 2 \mathrm{H}^{+}+\mathrm{O}^{2}+2 \mathrm{e}^{-}
$$

This kind of device, as-called "first generation biosensor", relates the concentration of the analyte or of the by-products from the enzymatic reactions to the electrical current developed on the electrode. In this case, the $\mathrm{H}_{2} \mathrm{O}_{2}$ production can be inspected under the $+0.7 \mathrm{~V} v s \mathrm{Ag} / \mathrm{AgCl}$ fixed potential or the $\mathrm{O}_{2}$ concentration can also be monitored under a $-0.7 \mathrm{~V} v s \mathrm{Ag} / \mathrm{AgCl}$ potential. To exploit the sensing phenomena at softer conditions, the second generation biosensors were developed using as main principle the use of electron carriers mediators that avoid the $\mathrm{O}_{2}$ dependence and diminish the influence of other electroactive species on the sensor response. The main disadvantage of this category is the lower stability in comparison to the first generation group. Thus, the third generation enzymatic sensors have been developed to overwhelm the previous limitations and to improve the response time to obtain quicker responses, in a mechanism in which the electron transfer takes place directly between the enzyme and the electrode. ${ }^{25}$ Despite most of the commercial glucose sensors relies on the enzymatic amperometric transduction, the use of enzymes possess some important advantages such as the low stability and high cost and, for this reason, some researchers have studied alternatives to develop non-enzymatic sensors even based on other electrochemical techniques. ${ }^{26}$ Among the analytes of interest in the food industry, the determination of azithromycin in animal source foods was studied by Jafari et al. ${ }^{25}$ due to the risk that this antibiotic can impose to consumers' health when present in excess in food products. The authors reported the use of differential pulse voltammetry as an electrochemical transduction technique to detect azithromycin, obtaining a limit of detection (LOD) equal to $0.1 \mathrm{nM}$ with high reproducibility $(n=8$; standard deviation of $2.5 \%)$. The research published by Montiel et al. ${ }^{27}$ using a disposable magnetic beads-based amperometric platform described the detection of the peanut allergenic proteins Ara h1 and Ara h2 at low LOD and with shorter assay time in comparison to the commercial kit based on the Enzyme-Linked Immunosorbent Assay (ELISA). Benedé et al. ${ }^{28}$ worked on the detection of ovomucoid, an egg white allergen, by employing a strategy of magnetic bio conjugated binding at the surface of a screen-printed carbon electrode under the influence of a fixed $-0.2 \mathrm{~V}$ (versus Ag pseudo reference electrode) potential. The calibration plot was linear in the concentration range from 0.3 to $25 \mathrm{ng} \cdot \mathrm{mL}^{-1}$ and this amperometric sensor was also more sensitive $\left(\mathrm{LOD}=0.1 \mathrm{ng} \cdot \mathrm{mL}^{-1}\right)$ than the commercial ELISA kit. Amperometric electronic noses also comprise a powerful category of sensors for monitoring food composition by means of the detection of volatile components. Gliszczyńska-Świgło \& Chmielewski ${ }^{29}$ highlighted that, in comparison to gas chromatography that is commonly used for the same purpose, the electronic noses possess as main advantages their simplicity, usually not requiring sample preparation, the low cost of each sample analysis, the short response time. Thus, they comprise an important tool for authentication of products that are commonly target of fraud, such as vegetable oils, meat products, seafood, beverages, fruits and honeys. ${ }^{28}$

\section{Impedimetric sensors}

Electrochemical Impedance Spectroscopy (EIS) is the technique that governs the transduction in impedimetric sensors. The EIS principle consist on applying a small sinusoidal potentiostatic perturbation on the electrochemical system under investigation in order to measure the impedance as a consequence of the resultant current (it is also possible to apply a current signal to obtain a resultant potential in the galvanostatic mode).$^{29}$ Assessing the components of the global impedance (real and imaginary impedance, phase angle, magnitude of impedance) and/or modelling the EIS data to an electrical equivalent circuit in order to obtain the values of resistors, capacitors and inductors, for instance, it is possible to presume the analyte presence/ concentration. ${ }^{29}$ Recently, our group developed impedimetric sensors for horsemeat adulteration screening and for glucose detection. The horsemeat immunosensor was capable to selectively detect the target analyte in buffered solution and in a solution prepared from commercial raw meat, presenting a LOD of $0.0004 \%$ (value that was lower than others reported in the literature by using spectrometry, chemiluminescence, fluorescence, voltammetry and amperometry). ${ }^{30}$ The glucose sensor, containing 3-aminophenylboronic acid as a probe, was also highly sensitive and presented a LOD equal to $8.53 \times 10^{-9} \mathrm{M}$. Besides its high sensitivity and low response towards other sugars (fructose and sucrose), this sensor presented as another advantage the capability to provide the output signal in few seconds $(4.0 \pm 0.6 \mathrm{~s})$ at optimal conditions. ${ }^{29}$ In both publications, we highlighted the use of screen-printed carbon electrodes a cheap material to construct the sensors, which make it cheap due to the easiness of its mass production. Furthermore, EIS represented an interesting transduction technique for online applications, besides the possibility to be carried out at a single frequency, which make it easy-to-perform. ${ }^{30,31}$ Das et $a l^{32}$ focused on developing an on-chip impedimetric sensor for identification and quantification of soap as an adulterant in milk. The authors pointed out that the traditional techniques used to detect fraud in milk, such as Near-Infrared and FTIR spectroscopy, High Performance Liquid Chromatography and Liquid Chromatography, are very laborious, require long processing times and demand expensive equipment despite the accuracy of the results. Other applications of EIS as a transduction method in sensors has also comprised the detection of allergens, microorganisms, monitoring of vegetable oils and fruits quality. ${ }^{33,34}$

\section{Conclusion}

In the context of consumers' vulnerability and protection, the food fraud detection has gained particular attention especially in the current decade. Aspects related to economic losses, consumer confidence, health risks, lifestyle and religious traditions motivated the scientific community to develop analytical techniques towards the recognition and quantification of fraudulent ingredients in many food products. The most exploited techniques commercially available mainly arise from spectrometric and chromatographic transduction mechanisms. However, some limitations, such as the sophisticated required apparatus, the time-consuming methodology and high cost of the equipment, hinder the viability of employing them in routine tests. As an alternative to the mentioned techniques, the electroanalytical 
assays represent an interesting opportunity in the food authentication scenario. High sensitive potentiometric sensors have been described in the literature to detect fraud especially in milk and alcoholic beverages by using electronic tongues in a fast and simple way. Despite advantageous because it is a non-destructive method (there is no concentration gradient of the electroactive species), the necessity of keeping the temperature very well controlled limits the potentiometric transduction to be used in in-situ analysis and hinders its reproducibility. Researches describing the use of amperometric sensors for inspection of fruits, honeys, dairy and meat products, for example, have shown low LOD towards analytes of interest with performances comparable to traditional techniques already available in the market. As a disadvantage, the common use of enzymes to mediate the amperometric response by producing intermediary electroactive species have led to the development of high cost and low stable sensors. Devices based on the EIS transduction technique have also been capable to detect low concentrated fraudulent ingredients in food products. Furthermore, since the impedimetric sensors lies on a non-destructive based technique (besides the possibility of using miniaturized electrodes), these devices are amenable for insitu and online measurements, which makes them very useful for the food quality industry. In this context, the use of electrochemical transduction in the field of food authentication has been shown to be promising and advantageous in comparison to other analytical techniques. The choice for the most appropriate electroanalytical assay most depends, though, on the target analyte, the aimed application (whether it is necessary to perform online in-situ measurements or not), the physicochemical conditions of the medium in which the sensing will be performed (temperature and presence of interfering species for example), the expected limit of detection/sensitivity and the required stability.

\section{Acknowledgments}

The authors thank to the Canada Excellence Research Chair in Photonics Innovations (CERCPI) and the Natural Sciences and Engineering Research Council of Canada (CRSNG).

\section{Conflicts of interests}

There is no conflict of interest.

\section{References}

1. Charlebois S, Summan A. A risk communication model for food regulatory agencies in modern society. Trends in Food Sci \& Tech. 2015;45(1):153-165.

2. Manning L, Soon JM. Food safety, food fraud, and food defense: a fast evolving literature. J Food Sci. 2016;81(4):823-834.

3. Grocery Manufacturers Association. Consumer product fraud and deterrence.

4. Hong E, Lee SY, Jeong JY, et al. Modern analytical methods for the detection of food fraud and adulteration by food category. J Sci Food Agric. 2017;97(12):3877-3896.

5. Nizar NNA, Zainal IH, Bonny SQ, et al. DNA and nanobiosensor technology for the detection of adulteration and microbial contamination in religious food. Prep Proces Relig Cult Foods. 2018;409-431.

6. Cohen LE, Felson M. Social change and crime rate trends: a routine activity approach. Amer Sociolog Rev. 1979;4(4):588-608.

7. Ruth SM, Huisman W, Luning PA. Food fraud vulnerability and its key factors. Trends in Food Sci \& Tech. 2017;67:70-75.
8. Charlebois S, Schwab A, Henn R, et al. Food fraud: an exploratory study for measuring consumer perception towards mislabeled food products and influence on self-authentication intentions. Trends in Food Sci \& Tech. 2016;50:211-218.

9. Pei X, Tandon A, Alldrick A, et al. The China melamine milk scandal and its implications for food safety regulation. Food Policy. 2011;36:412420 .

10. Falkheimer J, Heide M. Trust and Brand Recovery Campaigns in Crisis: Findus Nordic and the Horsemeat Scandal. Int J Strateg Commun. 2015;9:134-147.

11. Sheikha AFE. DNA Foil: novel technology for the rapid detection of food adulteration. Trends in Food Sci \& Techn. 2018; 86:544-542.

12. Danezis GP, Tsagkaris AS, Camin F, et al. Food authentication: techniques, trends \& emerging approaches. Trends in Anal Chem. 2016;85:123-132.

13. Hammond JL, Formisano N, Estrela P, et al. Electrochemical biosensors and nanobiosensors. Essays in Biochem. 2016;60:69-90.

14. MartinA, Vilela D, EscarpaA. Food analysis on microchip electrophoresis: an updated review. Electrophoresis. 2012;33:2212-2227.

15. Zhu C, Yang G, Li H, et al. Electrochemical sensors and biosensors based on nanomaterials and nanostructures. Anal Chem. 2015;87(1):230-249.

16. Naveen $\mathrm{MH}$, Gurudatt NG, Shim YB. Applications of conducting polymer composites to electrochemical sensors: a review. App Mat Today. 2017;9:419-433.

17. Wang $\mathrm{Y}, \mathrm{Xu} \mathrm{H}, \mathrm{Zhang} \mathrm{J}$, et al. Electrochemical sensors for clinical analysis. Sensors. 2008;8:2043-2081.

18. Trivedi UB, Lakshminarayana D, Kothari IL, et al. Potentiometric biosensor for urea determination in milk. Sens Act B: Chem. 2009;140(1):260-266.

19. Jiang H, Zhang M, Bhandari B, et al. Application of electronic tongue for fresh foods quality evaluation: a review. Food Rev Int. 2018;746-749.

20. Lvova L, Yaroshenko I, Kirsanov D, et al. Electronic tongue for brand uniformity control: a case study of apulian red wines recognition and defects evaluation. Sensors. 2018;18(8):2584.

21. Dias LG, Fernandes A, Veloso ACA, et al. Single-cultivar extra virgin olive oil classification using a potentiometric electronic tongue. Food Chem. 2014;160:321-329.

22. Holford TRJ, Davis F, Higson SPJ. Recent trends in antibody based sensors. Bios Bioelectron. 2012;34(1):12-24.

23. Thévenot DR, Toth K, Durst RA, et al. Electrochemical biosensors: recommended definitions and classification. Bios Bioelectron. 2001:16;121-131.

24. Clark LCJ, Lyons C. Electrode systems for continuous monitoring in cardiovascular surgery. Ann New York Acad Sci. 1962;102:29-45.

25. Jafari S, Dehghani $M$, Nasirizadeh N, et al. An azithromycin electrochemical sensor based on an aniline MIP film electropolymerized on a gold nano urchins/graphene oxide modified glassy carbon electrode. J Electroanal Chem. 2018;829:27-34.

26. Faria RAD, Iden H, Heneine LGD, et al. Non-enzymatic impedimetric sensor based on 3-aminophenylboronic acid functionalized screenprinted carbon electrode for highly sensitive glucose detection. Sensors. 2019;19:1686

27. Montiel VRV, Torrente-Rodríguez RM, Campuzano S, et al Simultaneous determination of the main peanut allergens in foods using disposable amperometric magnetic beads-based immunoassay platforms. Chemosens. 2016;4:1-11. 
28. Benedé S, Montiel RV, Povedano E, et al. Fast amperometric immunoplatform for ovomucoid traces determination in fresh and baked foods. Sens Act B: Chem. 2018;265:421-428

29. Gliszczyńska-Świgło A, Chmielewski J. Electronic nose as a tool for monitoring the authenticity of food. A review. Food Anal Methods. 2017;10:1800-1816.

30. Faria RAD, Heneine LGD, Matencio T, et al. Faradaic and non-faradaic electrochemical impedance spectroscopy as transduction techniques for sensing applications. Int J Bios Bioelectron. 2019;5(1):29-31.

31. Faria RAD, Iden $\mathrm{H}$, Bharucha $\mathrm{E}$, et al. A new tool for the detection of horsemeat adulteration in raw meat. J Bios Bioelectron. 2018;9(4):1-7
32. Das C, Chakraborty S, Karmakar A, et al. On-chip detection and quantification of soap as an adulterant in milk employing Electrical Impedance Spectroscopy. Int Symp Dev, Circ Systems (ISDCS). 2018;14.

33. Neethirajan S, Weng $X$, Tah A, et al. Nano-biosensor platforms for detecting food allergens - new trends. Sens Bio-Sens Res. 2018;18:1330 .

34. Grossi M, Riccò B. Electrical Impedance Spectroscopy (EIS) for biological analysis and food characterization: a review. J Sens Sens Syst . 2017;6:303-325. 